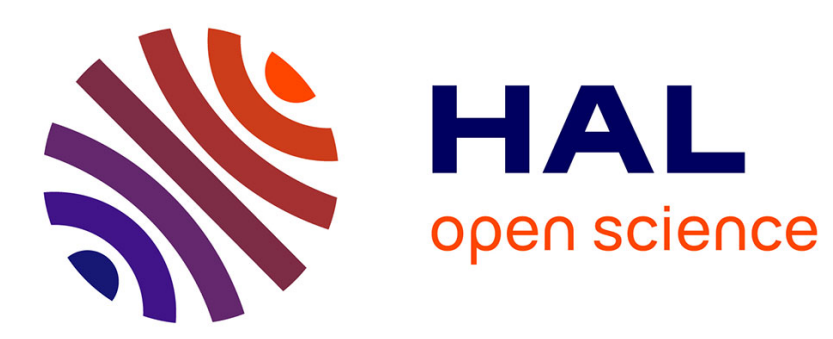

\title{
Simulation and sensitivity analysis of sensors network for cardiac monitoring
}

Yaël Kolasa, Thierry Bastogne, Jean-Philippe Georges

\section{To cite this version:}

Yaël Kolasa, Thierry Bastogne, Jean-Philippe Georges. Simulation and sensitivity analysis of sensors network for cardiac monitoring. 8th International Digital Health Conference, DH2018, Apr 2018, Lyon, France. hal-01925649

\section{HAL Id: hal-01925649 https://hal.science/hal-01925649}

Submitted on 16 Nov 2018

HAL is a multi-disciplinary open access archive for the deposit and dissemination of scientific research documents, whether they are published or not. The documents may come from teaching and research institutions in France or abroad, or from public or private research centers.
L'archive ouverte pluridisciplinaire HAL, est destinée au dépôt et à la diffusion de documents scientifiques de niveau recherche, publiés ou non, émanant des établissements d'enseignement et de recherche français ou étrangers, des laboratoires publics ou privés. 


\section{Simulation and sensitivity analysis of sensors network for cardiac monitoring}

\author{
Yaël Kolasa \\ Cybernano \\ Villers-lès-Nancy, France \\ ykolasa@cybernano.eu
}

\author{
Thierry Bastogne \\ U. Lorraine, CNRS, CRAN, UMR 7039 \\ INRIA BIGS \\ Vandouvre-lès-Nancy, France
}

\author{
Jean-Philippe Georges \\ U. Lorraine, CNRS, CRAN, UMR 7039 \\ Vandœuvre-lès-Nancy, France
}

\section{Network Modeling (Omnet++)}

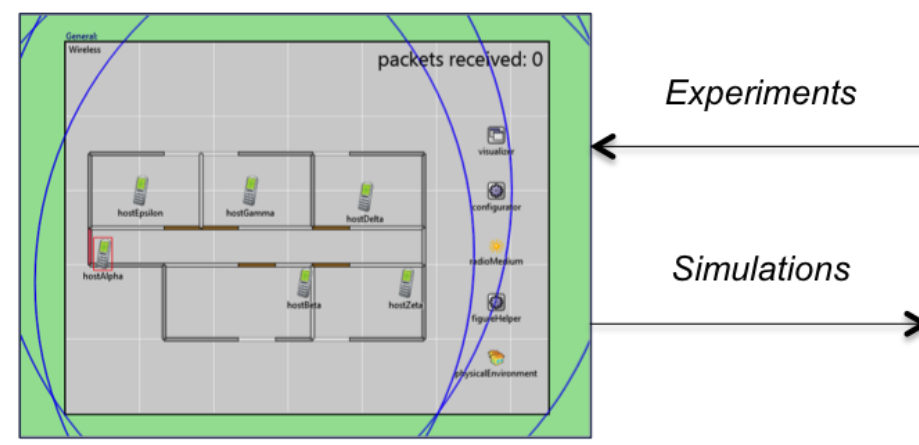

Sensitivity Analysis (Matlab)

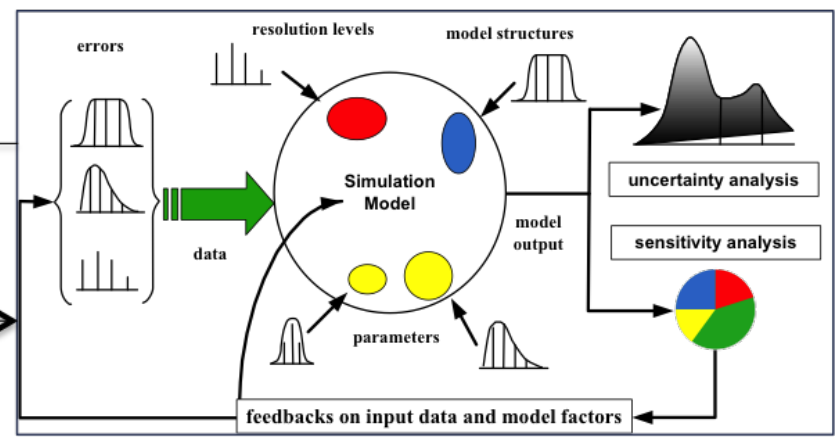

Figure 1. Design of numerical experiments for the sensitivity analysis of sensors networks

\begin{abstract}
This study's aim was to model and simulate a wireless sensor network and to propose a two-step sensitivity analysis for a targeted application related to home cardiac monitoring. After an initial phase of research to design the appropriate network simulator implemented in Omnet++, 13 simulation parameters have been selected to test their criticality. The sensitivity analysis relies on two consecutive steps carried out in Matlab: a screening phase (Plackett-Burman design) and a global sensitivity analysis (Space-fiiling design). Two output variables are considered: the number of packets received by the sink and the reception cache hit percentage. Four critical simulation parameters have been identified: the message length, bit rate, the background noise power and the energy detection of the radio receiver. In perspective, this sensitivity analysis will be included as a component of a Quality-by-Design approach of network development. This contribution is the early stage result obtained by Y. Kolasa during his $\mathrm{MsC}$ and $\mathrm{PhD}$ thesis (begun end of 2017) [2].
\end{abstract}

Permission to make digital or hard copies of part or all of this work for personal or classroom use is granted without fee provided that copies are not made or distributed for profit or commercial advantage and that copies bear this notice and the full citation on the first page. Copyrights for thirdparty components of this work must be honored. For all other uses, contact the owner/author(s).

DH'2018, April 2018, Lyon, France

(c) 2018 Copyright held by the owner/author(s).

ACM ISBN 978-x-xxxx-xxxx-x/YY/MM

https://doi.org/10.1145/nnnnnnn.nnnnnnn
CCS Concepts - Computer systems organization $\rightarrow$ Simulation; Sensors; $\bullet$ Networks $\rightarrow$ Network reliability; Security; • Statistics $\rightarrow$ Robustness Analysis;

Keywords Internet of Things; Simulation; Sensitivity Analysis; Cardiac Monitoring

\section{ACM Reference Format:}

Yaël Kolasa, Thierry Bastogne, and Jean-Philippe Georges. 2018. Simulation and sensitivity analysis of sensors network for cardiac monitoring. In Proceedings of 8th ACM International Digital Health Conference (DH'2018). ACM, New York, NY, USA, 2 pages. https: //doi.org/10.1145/nnnnnnn.nnnnnnn

\section{Introduction}

This study comes from a double context, the high-throughput analysis in preclinical cardiotoxicity tests and the growing use of smart or connected objects to monitor patients' health. In both cases, the goal is to transmit cardiac signals for remote processing. These last years, the improvement of measuring systems in pharmaceutical laboratories allowed to gather more accurate and numerous data during preclinical analysis. This increase in data size has two direct consequences : problem of storage, and sharing over internet $[1,4,6]$. Furthermore, some of those can contain sensitive data for which confidentiality must be ensured. In parallel, more and more smart objects are available and allow to monitor daily cardiorespiratory activity over long period of time, longer than traditional ECG (10s) or Hölter ECG (24h). Those huge generated files beget transfer problems 
to which are added network connectivity constraints. Both times, files' size can sire too long transfer times, files' potential corruption, and a poor users' experience quality. In order to ensure a faultless quality of service, we propose thereafter an innovative approach combining network simulation and sensibility analysis.

\section{Network Modeling}

\subsection{A Study Case}

The study case was to simulate a network of five sensors. One of them was moving, and was the only emitter of messages. Another one was the sink of the network, the only target, which was able to acknowledge received messages. The other three were retransmitters, used to forward the messages if they received them. The emitter moves straight through a modeled flat with different materials and ensuing different disturbances.

\subsection{Omnet++ Implementation}

This case was implemented in Omnet++ with the help of the library INET, which provides more realistic elements to build a simulation with. It integrates widely used TCP and UDP protocols, support for sensor networks, energy consumption and management, interferences, path loss, etc.

\subsection{Model \& Simulation Outputs and Parameters}

Two output variables (quality attributes) were examined. The first one was the number of packets received by the sink at the end of each simulation. The second output is the reception cache hit percentage. 13 network parameters have to be tested: the message length, the send interval, radio types and associated parameters, battery power, mac type, the use (or not) of acknowledge receipt of a packet, carrier frequency, energy detection and associated parameters, bit rate, max queue size, header bit length, path loss type and antenna type. They were selected to have a wide range of action on the sensors' capacity to interact with their environment.

\section{Sensitivity Analysis}

The sensitivity analysis was split into two phases. The first one aims at screening the most active parameters through a minimal number of simulations while in a second step a global sensitivity analysis was carried out to rank the total effects of the parameters selected after the screening study.

\subsection{Screening of Parameters}

A Plackett-Burman design of experiments was used to implement the preliminary selection of active parameters [3].

\subsection{HDMR-ANOVA}

Once the most active simulation factors have been identified, a Sobol' sequence was implemented to generate the simulated data we need to estimate the sensitivity indices by an
Analysis of Variance approach based on a High Dimensional Model Representation (HDMR-ANOVA) [5].

\subsection{Matlab Implementation}

Matlab was used to generate a Sobol' sequence of experiments and Omnet++ was launched via a batch file specifically created to run each simulation of the experimental design.

\section{Results}

For the number of packets received, two critical parameters were identified: the message length and the bit rate. For the reception cache hit percentage, the first two most critical factors are the background noise power and the energy detection of the radio receiver.

\section{Conclusion}

A prototype sensitivity analysis of a sensors network guided by simulations has been proposed. Preliminary results have demonstrated its practical feasibility by combining two simulation environments: Omnet++ and Matlab. The proposed technique allows to quickly identify the most critical parameters impacting the whole quality of service of the network. This $\mathrm{PhD}$ thesis will be carried out in the context of the Hopital virtuel de Lorraine to better account for the constraints of the medical context. The main objective is to develop a holistic and safe engineering approach of medical sensors networks following the recents works on Quality-by-Design [2] and by adapting it to remote cardiac monitoring.

\section{References}

[1] Mohammed Aledhari and Fahad Saeed. 2015. Design and Implementation of Network Transfer Protocol for Big Genomic Data. In 2015 IEEE INTERNATIONAL CONGRESS ON BIG DATA - BIGDATA CONGRESS 2015 (IEEE International Congress on Big Data), Barbara, C and Khan, L (Ed.). IEEE; IEEE Comp Soc; Serv Comp; Serv Soc; Comp Cloud; Serv Comp; BIG Data; Hp; IBM; ERICSSON; SAP; IBM Res; HUAWEI; OMG Object Management Grp; IEEE Cloud Comp; Business Proc Integrat \& Management; IT Profess; Intl Journal Web Serv Res; Comp Now Access Discover Engage; IEEE Transact Serv Comp, 281-288. https://doi.org/\{10.1109/BigDataCongress.2015.47\} IEEE International Congress on Big Data, New York, NY, JUN 27-JUL 02, 2015.

[2] Thierry Bastogne. 2017. Quality-by-design of nanopharmaceuticals A state of the art. Nanomedicine: Nanotechnology, Biology and Medicine 13, 7 (2017), 2151-2157.

[3] G. A. Lewis, D. Mathieu, and R. Phan-Tan-Luu. 2005. Pharmaceutical Experimental Design. Marcel Dekker.

[4] V. Marx. 2013. The Big Challenges of Big Data. Nature 498 (2013), 255-260.

[5] A. Saltelli, M. Ratto, T. Andres, F. Campolongo, J. Cariboni, D. Gatelli, M. Saisana, and S. Tarantola. 2008. Global Sensitivity Analysis - The Primer. Wiley.

[6] Brian Tierney, Ezra Kissel, Martin Swany, and Eric Pouyoul. 2012. Efficient Data Transfer Protocols for Big Data. In 2012 IEEE 8TH INTERNATIONAL CONFERENCE ON E-SCIENCE (E-SCIENCE) (Proceeding IEEE International Conference on e-Science (e-Science)). IEEE; IEEE Comp Soc. IEEE 8th International Conference on E-Science (e-Science), Chicago, IL, OCT 08-12, 2012. 